\title{
Retraction Note to: Prevalence of Nontuberculous Mycobacterial Disease in the Changchun District of China
}

\author{
Wang Chunfang $^{1} \cdot$ Ren Jihong $^{1} \cdot$ Wang Yu ${ }^{2} \cdot$ Zhao Yunhong $^{2} \cdot$ Sun Xuejuan ${ }^{2} \cdot$ Jiang Xiuyun $^{1,3} \cdot$ Wang Chunfeng $^{1}$
}

Published online: 4 October 2021

(c) Springer Science+Business Media, LLC, part of Springer Nature 2021

\section{Retraction Note to: \\ Current Microbiology (2021) 78:1643-1647 \\ https://doi.org/10.1007/s00284-021-02422-y}

The Publisher and Editor have retracted this article [1] because it was inadvertently accepted and published owing to an administrative error. The Publisher apologizes to the authors and to the Editors-in-Chief.

None of the authors agree to this retraction.

\section{Reference}

1. Chunfang W, Jihong R, Yu W et al (2021) Prevalence of nontuberculous mycobacterial disease in the Changchun district of China. Curr Microbiol 78:1643-1647. https://doi.org/10.1007/ s00284-021-02422-y

The original article can be found online at https://doi.org/10.1007/ s00284-021-02422-y.

Sun Xuejuan

15943013355@163.com

$\triangle$ Jiang Xiuyun

jiangxiuyun@jlau.edu.cn

$\triangle$ Wang Chunfeng

wangchunfeng@jlau.edu.cn

1 College of Animal Medicine, College of Animal Science and Technology, College of Life Science, Jilin Provincial Engineering Research Center of Animal Probiotics and Key Laboratory of Animal Production and Product Quality Safety of Ministry of Education, Jilin Agricultural University, Changchun 130118, China

2 Changchun Infectious Disease Hospital, Changchun 130000, China

3 Changchun Sci-Tech University, Changchun 130118, China
Publisher's Note Springer Nature remains neutral with regard to jurisdictional claims in published maps and institutional affiliations. 